\title{
Motives, numerical equivalence, and semi-simplicity
}

\section{Uwe Jannsen}

Mathematisches Institut, Weyertal 86-90, W-5000 Köln 41, Federal Republic of Germany

Oblatum 12-VII-1991

In this note we show that the category of motives, as defined via algebraic correspondences modulo an adequate equivalence relation, is a semi-simple abelian category if - and in fact, only if - the relation is numerical equivalence. Contrary to general belief Grothendieck's standard conjectures are not needed for this.

Let $k$ be a field, and consider smooth projective varieties over $k$, called varieties for short in the following. Fix a field $F$ of characteristic zero, and an adequate equivalence relation $\sim$ on $F$-linear algebraic cycles ( $\mathrm{cf}[\mathrm{K} 2] \S 2$; in short, pull-back, push-forward and intersection of cycles is always (well-)defined modulo $\sim$ ). For a variety $X$ and an integer $j \geqq 0$, denote by $A^{j}(X)=A^{j}(X, F)$ the group of $F$-linear algebraic cycles of codimension $j$ on $X$ modulo $\sim$. If $X$ has connected components $X_{1}, \ldots, X_{n}$ of dimensions $d_{1}, \ldots, d_{n}$, and if $Y$ is a second variety, we put

$$
A^{\operatorname{dim}(X)+\mathrm{r}}(X \times Y)=\bigoplus_{i=1}^{n} A^{d_{i}+r}\left(X_{i} \times Y\right)
$$

for $r \in \mathbb{Z}$; similar notations apply to other products. Then one has a bilinear composition law

$$
A^{\operatorname{dim}\left(X_{1}\right)+r}\left(X_{1} \times X_{2}\right) \times A^{\operatorname{dim}\left(X_{2}\right)+s}\left(X_{2} \times X_{3}\right) \rightarrow A^{\operatorname{dim}\left(X_{1}\right)+r+s}\left(X_{1} \times X_{3}\right)
$$

by sending $(f, g)$ to

$$
g \circ f=\left(p_{13}\right)_{*}\left(p_{12}^{*} f \cdot p_{23}^{*} g\right),
$$

where $p_{i j}: X_{1} \times X_{2} \times X_{3} \rightarrow X_{i} \times X_{j}$ is the projection. This composition law is associative in an obvious sense. In particular, $A^{\operatorname{dim}(X)}(X \times Y)$ is a ring.

The category $M_{k}$ of motives w.r.t. $\sim$ can be defined as follows (cf. [K2] Sect. 1; the definition here is not the one usually taken in the literature, but is easily seen to be equivalent to it, cf. the formula in the middle of p. 57 in loc. cit.).

Objects of $M_{k}$ are triples $(X, p, m)$, where $X$ is a variety, $p \in A^{\operatorname{dim}(X)}(X \times X)$ is a projector (i.e., $p^{2}=$ p), and $m$ is an integer. The morphism sets are defined by putting

$$
\operatorname{Hom}((X, p, m),(Y, q, n))=q A^{\operatorname{dim}(X)-m+n}(X \times Y) p
$$


and the composition of morphisms is the one induced by the composition law above.

Remark. In the notation of loc. cit., $(X, p, m)=M(m)$ for the effective motive $M=(X, p)$.

$M_{k}$ is an $F$-linear, pseudo-abelian category, cf. [K2] Sect. 5.

Theorem 1. The following properties are equivalent.

a) $M_{k}$ is a semi-simple abelian category.

b) $A^{\operatorname{dim}(X)}(X \times X)$ is a finite-dimensional, semi-simple $F$-algebra for every variety $X$.

c) The relation $\sim$ is numerical equivalence.

Proof. Recall that, obviously, numerical equivalence is the coarsest adequate equivalence relation. a) $\rightarrow \mathrm{c})$ : Let $1=(\operatorname{Spec} k, i d, 0)$ be the identity object and let $X$ be an irreducible variety. Since $\operatorname{End}(1)=F$ is a field, 1 is an irreducible object. Hence every non-trivial morphism $f: 1 \rightarrow(X, i d, j)$ is a monomorphism, and by a) there is a morphism $g:(X, i d, j) \rightarrow 1$ with $g \circ f=i d$. Translated back to algebraic cycles this means: for every non-zero $f \in A^{j}(X)$ there is a $g \in A^{\operatorname{dim}(X)-j}(X)$ with $p_{*}(f \cdot g)=1 \in F=A^{\circ}$ (Spec $\left.k\right)$, where $p: X \rightarrow$ Spec $k$ is the structural morphism. But via the indicated identification $F=A^{\circ}(\operatorname{Spec} k), p_{*}(f \cdot g)$ is just the intersection number $\langle f \cdot g\rangle$ of $f$ and $g$.

c) $\rightarrow$ b): Let $\sim$ be numerical equivalence, and fix a Weil cohomology $X \mapsto H(X)$, for example the one given by the étale cohomology groups $H^{i}\left(X \times_{k} k_{s}, \mathbb{Q}_{l}\right)$, where $k_{s}$ is a separable closure of $k$, and $l$ is a prime number invertible in $k$. Denote the corresponding homological equivalence by $\sim$ ', and the cycle groups modulo $\sim^{\prime}$ by $B^{j}(X)$.

It is well-known that $A^{j}(X)$ is a finite-dimensional $F$-vector space for all $X$ and $j$, and that one has an isomorphism $A^{j}(X, \mathbb{Q}) \otimes_{\mathbb{Q}} F \simeq A^{j}(X, F)$, cf. [K1] Thm. 3.5. In particular, the Jacobson radical $J$ of $A^{\operatorname{dim}(X)}(X \times X, F)$ is obtained from the one of $A^{\operatorname{dim}(X)}(X \times X, \mathbb{Q})$ by base extension to $F$, and it suffices to show $J=0$ over any field $F$. Let us consider $F=\mathbb{Q}_{l}$. The following formula is well-known, see, e.g. [K1] 1.3.6.

Lemma 1. Let $f \mapsto^{t} f, B^{\operatorname{dim}(X)}(X \times X) \rightarrow B^{\operatorname{dim}(X)}(X \times X)$, be the transposition, induced by interchanging the factors of $X \times X$. Then for $f, g \in B^{\operatorname{dim}(X)}(X \times X)$ one has

$$
\langle f \cdot t\rangle=\sum_{i=0}^{2 \operatorname{dim}(X)}(-1)^{i} \operatorname{Tr}_{i}(f \circ g),
$$

where $\operatorname{Tr}_{i}(f)$ is the trace of $f$ acting on $H^{i}(X)$.

Now consider the surjection of finite-dimensional $\mathbb{Q}_{l}$-algebras

$$
S: B=B^{\operatorname{dim}(X)}(X \times X) \rightarrow A^{\operatorname{dim}(X)}(X \times X)=A
$$

and let $J^{\prime}$ be the Jacobson radical of $B$. Since $B / J^{\prime}$ is semi-simple and this carries over to quotients, and since $S\left(J^{\prime}\right)$ is a nilpotent two-sided ideal in $A$, we must have $S\left(J^{\prime}\right)=J$. Now let $f \in J$ and choose a lifting $f^{\prime} \in J^{\prime}$ under $S$. For every $g \in B, f^{\prime} \circ g$ lies in $J^{\prime}$ and hence is nilpotent. The same is true for its image in $\operatorname{End}_{\mathbb{Q}_{1}}\left(H^{i}(X)\right)$, so we must have $\operatorname{Tr}_{i}\left(f^{\prime} \circ g\right)=0$. Since this is true for all $i \geqq 0$, we conclude that 
$\left\langle f^{\prime} \cdot t^{t}\right\rangle=0$ for all $g \in B$ by the lemma. By definition this means that $f^{\prime}$ lies in the kernel of $S$, i.e., that $f=0$.

b) $\rightarrow$ a): This is certainly well-known and only included for the convenience of the reader. First we deduce from $b$ ) that $\operatorname{End}_{M_{k}}(M)$ is a finite-dimensional, semi-simple $F$-algebra for every direct factor $M$ of $h(X)=(X, i d, 0)$ and hence for every object $M$ of $M_{k}$. In fact, if $J$ is a nilpotent two-sided ideal of $\operatorname{End}(M)$, and if $h(X)=M \oplus N$, then

$$
\left(\begin{array}{cc}
J & J \circ \operatorname{Hom}(N, M) \\
\operatorname{Hom}(M, N) \circ J & \operatorname{Hom}(M, N) \circ J \circ \operatorname{Hom}(N, M)
\end{array}\right)
$$

is a nilpotent two-sided ideal of $\operatorname{End}(h(X))$. The rest of the conclusion is formulated in a lemma:

Lemma 2. Let $C$ be an F-linear, pseudo-abelian category such that $\operatorname{End}(M)$ is a finite-dimensional, semi-simple F-algebra for every object $M$ of $C$. Then $C$ is a semi-simple abelian category.

Proof. By Wedderburn's theorem, $\operatorname{End}(M)$ is a product of full matrix algebras over skewfields. This shows that $M$ is indecomposable if and only if $\operatorname{End}(M)$ is a skewfield. Since obviously every object is a finite sum of indecomposables, it suffices to show that for two indecomposables $M$ and $N$ either $\operatorname{Hom}(M, N)=0$ or $M$ and $N$ are isomorphic. Indeed, then $C$ is equivalent to a sum, indexed by the isomorphism classes of indecomposables (= irreducibles), of categories which are equivalent to categories of vector spaces over skewfields.

Let $M$ and $N$ be indecomposable. If $\operatorname{Hom}(M, N) \neq 0$, then the composition

$$
\begin{aligned}
\operatorname{Hom}(N, M) \times \operatorname{Hom}(M, N) & \rightarrow \operatorname{End}(M) \\
(f, g) & \mapsto f \circ g
\end{aligned}
$$

is non-zero, since otherwise

$$
\left(\begin{array}{cc}
0 & 0 \\
\operatorname{Hom}(M, N) & 0
\end{array}\right) \subseteq\left(\begin{array}{cc}
\operatorname{End}(M) & \operatorname{Hom}(N, M) \\
\operatorname{Hom}(M, N) & \operatorname{End}(N)
\end{array}\right)=\operatorname{End}(M \oplus N)
$$

was a non-trivial nilpotent two-sided ideal. Take $(f, g)$ with $f \circ g \neq 0$. As $\operatorname{End}(M)$ is a skewfield, $f \circ g$ is invertible and $(f g)^{-1} \circ f$ is a left inverse of $g . N$ being indecomposable, $g$ must be an isomorphism. q.e.d.

Corollary 1. Denote by $A_{\text {num }}^{\cdot(-)}$ the cycle groups modulo numerical equivalence and by $A_{\text {hom }}(-)$ those modulo homological equivalence - with respect to some fixed Weil cohomology $X \mapsto H(X)$. If, for a variety $X$, the Künneth components $\pi^{j} \in H^{2 \operatorname{dim}(X)-j}(X) \otimes H^{j}(X)$ of the diagonal are algebraic (cf. [K1] Sect. 2), then the kernel of the surjective ring homomorphism

$$
S: B=A_{\text {hom }}^{\operatorname{dim}(X)}(X \times X) \rightarrow A_{\mathrm{num}}^{\operatorname{dim}(X)}(X \times X)=A
$$

is the Jacobson radical of $B$, and is a nilpotent ideal.

Proof. By the proof of c) $\rightarrow$ b) of Theorem 1, the radical $J^{\prime}$ of $B$ is contained in the kernel of $S$. Now, by definition of the $\pi^{j}$ we have

$$
\operatorname{Tr}_{i}\left(f \circ \pi^{j}\right)=\delta_{i j} \operatorname{Tr}_{j}(f)
$$


for every $f \in B$, where $\delta_{i j}$ is the Kronecker symbol and $\operatorname{Tr}_{i}$ is as in Lemma 1. If $f$ is numerically equivalent to zero, and if the $\pi^{j}$ are algebraic, then the above formula and Lemma 1 show that $\operatorname{Tr}_{i}(f)=0$ for all $i \geqq 0$. The same is true for all powers of $f$, which implies that for all $i \geqq 0$ the endomorphism $f_{i}$ of $H^{i}(X)$ induced by $f$ has zero eigenvalues, thus is nilpotent. By definition, the cycle map

$$
B \hookrightarrow H^{2 \operatorname{dim}(X)}(X \times X)=\underset{i \geqq 0}{\oplus} \text { End } H^{i}(X)
$$

is an injective ring homomorphism, so that $f$ is nilpotent in $B$.

Up to now we did not have to worry about the coefficient field $A$ of $H$. It must be an $F$-algebra, for the groups $A_{\text {hom }}(-)=A_{\text {hom }}(-, F)$ to be defined. First consider the case $F=A$. Then $B$ is a finite-dimensional $A$-algebra, and the fact that every element in $\operatorname{Ker} S$ is nilpotent implies that $\operatorname{Ker} S$ is a nilpotent ideal and contained in the radical. In the general case it is conjectured but not known (for $\operatorname{char}(k)>0)$ that $B$ is finite-dimensional over $F$. However, by the commutative diagram

$$
\begin{gathered}
S_{A}: A_{\mathrm{hom}}^{\operatorname{dim}(X)}(X \times X, A) \rightarrow A_{\mathrm{num}}^{\operatorname{dim}(X)}(X \times X, A) \\
\uparrow \\
\uparrow \\
S_{F}: A_{\mathrm{hom}}^{\operatorname{dim}(X)}(X \times X, F) \rightarrow A_{\text {num }}^{\operatorname{dim}(X)}(X \times X, F),
\end{gathered}
$$

in which the left vertical arrow obviously is injective, we still conclude that $\operatorname{Ker} S_{F}$ is a nilpotent ideal and hence contained in the radical since this is true for $\operatorname{Ker} S_{A}$.

Corollary 2. If the Künneth components of the diagonal (w.r.t. some fixed Weil cohomology $X \mapsto H(X))$ are algebraic for every variety $X$ over $k$, then the category $M_{k}$ of motives with respect to numerical equivalence is a semi-simple F-linear Tannakian category (cf. [SR] III 3.2.1, [D] 2.8., 1.2).

Proof. For every adequate equivalence relation $\sim$, the category $M_{k}$ with respect to $\sim$ is an $F$-linear tensor category ([DM] 1.1; " $\otimes$-catégorie ACU" in the terminology of $[\mathrm{SR}]$ ), the tensor law being given by

$$
(X, p, m) \otimes(Y, q, n)=(X \times Y, p \times q, m+n),
$$

the commutativity and associativity constraints being induced by the obvious isomorphisms $X \times Y \neg Y \times X$ and $X \times(Y \times Z) \neg(X \times Y) \times Z$, respectively. A unit object is given by $1=(\operatorname{Spec} k, i d, 0)$. Moreover, $M_{k}$ is rigid ([DM] 1.7), with the internal Hom in the case of an irreducible $X$ defined by

$$
\underline{\operatorname{Hom}}((X, p, m),(Y, q, n))=\left(X \times Y,{ }^{t} p \times q, \operatorname{dim}(X)-m+n\right) \text {. }
$$

Let now $\sim$ be numerical equivalence. Then $M_{k}$ is semi-simple abelian by Theorem 1 , so by [D] it only needs a fibre functor with values in some extension field $F^{\prime}$ of $F=$ End(1) to make $M_{k}$ into a Tannakian category.

But it is well-known that such a fibre functor cannot exist (cf. [DM] p. 203): under the above normalizations the rank (in the sense of [DM] p. 113) of a motive $h(X)=(X, i d, 0)$ can be negative, while in the presence of a fibre functor it should be non-negative, as the dimension of a vector space (loc. cit., formulas after Prop. 1.11). In fact, one easily computes $r k(h(X))=\langle\Delta \cdot \Delta\rangle$ for the diagonal $\Delta \varsigma X \times X$, and this can be positive as well as negative, cf. Lemma 1 . 
Now assume that for every variety $X$ the Künneth components $\pi^{j}=\pi_{X}^{j}$ of the diagonal are algebraic, and denote their images in $A_{\mathrm{num}}^{\mathrm{dim} X}(X \times X)$ again by $\pi^{j}$. Note that the $\pi^{j}$ are central idempotents, with $\pi^{j}$ orthogonal to $\pi^{i}$ for $i \neq j$. Thus every motive $M$ gets a $\mathbb{Z}$-grading by putting

$$
(X, p, m)^{r}=\left(X, p \pi^{r+2 m}, r\right),
$$

and this grading is respected by all morphisms. Moreover, the commutativity constraints

$$
\psi: M \otimes N \Im N \otimes M
$$

can be written as $\psi=\oplus_{r, s} \psi^{r, s}$ with

$$
\psi^{r, s}: M^{r} \otimes N^{s} \neg N^{s} \otimes M^{r} .
$$

If we modify the constraints by defining

$$
\psi_{\text {new }}=\oplus_{r, s}(-1)^{r s} \psi^{r, s},
$$

then the computation leading to Lemma 1 easily shows

$$
r k((X, p, m))=\sum_{i \geqq 0} \operatorname{dim}_{\Lambda} p H^{i}(X) \geqq 0
$$

for every motive $(X, p, m)$ in $M_{k}$. By Deligne's theorem 7.1 in [D], $M_{k}$ is Tannakian.

Remarks. 1) The assumption of Corollary 1 is fulfilled if $\operatorname{dim} X \leqq 2([M])$ or if $X$ is an abelian variety (by results of Lieberman, cf. [K] Sect. 2 appendix), and for all $X$ if $k$ is (contained in the algebraic closure of) a finite field ([KM]). Moreover, if the assumption holds for $X$ and $Y$, it also holds for $X \times Y$, since

$$
\pi_{X \times Y}^{j}=\sum_{r+s=j} \pi_{X}^{r} \otimes \pi_{Y}^{s}
$$

by the Künneth formula.

2) In particular, the assumption of Corollary 2 is fulfilled if $k$ is an algebraic extension of a finite field. Moreover, the result of Corollary 2 holds for every subcategory $C$ of $M_{k}$ generated (as an abelian tensor category) by 1(1) and a family of varieties $\left(X_{i} \mid i \in I\right)$ such that the Künneth components of the diagonal are algebraic for the $X_{i}$. Note that $C$ consists of the motives $(X, p, m)$ where $X$ is a product of some of the $X_{i}$. In particular, the category of motives generated by abelian varieties is a Tannakian category over any field $k$.

3) Lemma 2 should replace Lemma 6.6 in $[D M]$ which is false: let $\mathbb{Q}[\varepsilon]=\mathbb{Q} \oplus \mathbb{Q} \varepsilon$ with $\varepsilon^{2}=0$ be the $\mathbb{Q}$-algebra of dual numbers. Then the category $C$ of finitely generated free $\mathbb{Q}[\varepsilon]$-modules is a $\mathbb{Q}$-linear pseudo-abelian category with a faithful Q-linear functor into $\mathrm{Vec}_{\mathbb{Q}}$ (the forgetful functor), and every indecomposable object of $C$ is simple, but $C$ is not an abelian category. In fact, the endomorphism $\varepsilon$ of $\mathbb{Q}[\varepsilon]$ has no kernel. The same wrong argument seems to appear in Saavedra's proof of [SR] VI Th. 4.2.2.

4) Under the assumption of Corollary 1 , one can associate cohomology groups $H(M)$ to every motive $M=(X, p, m)$ w.r.t. numerical equivalence, such that $H(M)$ 
is well-defined up to non-canonical isomorphism. In fact, one can lift $p$ to an idempotent $p^{\prime}$ in $A_{\mathrm{hom}}^{\operatorname{dim}(X)}(X \times X)$ (cf. the lemma in [M] 7.3 bis) and can put

$$
H^{i}(M)=p^{\prime} H^{i+2 m}(X) \text {. }
$$

Since another lifting $p^{\prime \prime}$ of $p$ differs from $p^{\prime}$ by conjugation with a unit in $A_{\text {hom }}^{\operatorname{dim}(X)}(X \times X)$ (loc. cit.), $p^{\prime \prime} H^{i+2 m}(X)$ is isomorphic to $p^{\prime} H^{i+2 m}(X)$.

Acknowledgement. The main result of this paper was obtained during a stay at the CRM (Bellaterra, Spain). I thank the institute for the invitation and pleasant stay.

\section{References}

[D] Deligne, P.: Catègories tannakiennes. The Grothendieck Festschrift. vol. II, pp. 111-195, Boston: Birkhäuser 1990

[DM] Deligne, P., Milne, J.S.: Tannakian categories. In: Hodge cycles, motives, and Shimura varieties. (Lect. Notes Math., Vol. 900, pp. 101-228) Berlin Heidelberg New York: Springer 1982

[KM] Katz, N., Messing, W.: Some consequences of the Riemann hypothesis for varieties over finite fields. Invent. math. 23, 73-77 (1974)

[K1] Kleiman, S.L.: Algebraic cycles and the Weil conjectures, Dix exposès sur la cohomologie des schemas. Giraud, J. (ed.) pp. 359-386. Amsterdam: North-Holland, 1968

[K2] Kleiman, S.L.: Motives, algebraic geometry, Oslo 1970. Oort, F. (ed.), pp. 53-82. Groningen: 1972

[M] Murre, J.P.: On the motive of an algebraic surface. J. Reine Angew. Math. 409, 190-204 (1990)

[SR] Saavedra Rivano, N.: Catègories Tannakiennes. (Lect. Notes Math., vol. 265) Berlin Heidelberg New York: Springer 1972 